\title{
Espiritualidade, Religiosidade, Distress e Qualidade de Vida em Pacientes Oncológicos
}

Spirituality, Religiosity, Distress and Quality of Life in Oncological Patients Espiritualidad, Religiosidad, Distress y Calidad de Vida en Pacientes Oncológicos

\author{
Getúlio Yuzo Okuma \\ Maria Fernanda Marcusso Manhães \\ Renata Nunes Pedras \\ Isadora Miranda Azevedo \\ Edvane Birelo Lopes De Domenico \\ Universidade Federal de São Paulo (UNIFESP)
}

Cristiane Decat Bergerot ${ }^{1}$

City of Hope Comprehensive Cancer Center, Department of Medical Oncology \& Experimental Therapeutics

\section{Resumo}

O presente estudo teve o objetivo de avaliar a relação entre o distress, a qualidade de vida e a prática espiritual e religiosa (ER) em pacientes recém-diagnosticados com câncer, na primeira linha de tratamento quimioterápico. Trata-se de um estudo transversal, que avaliou 100 pacientes quanto ao distress (Termômetro de Distress), qualidade de vida (Functional Assessment of Chronic Illness Therapy - General) e espiritualidade (Functional Assessment of Chronic Illness Therapy - Spiritual Well-Being Scale e Escala de Religiosidade da Universidade de Duke). Os resultados sugerem que a prática ER é uma importante estratégia no enfrentamento do diagnóstico e tratamento do câncer, estando associada significativamente a um menor nível de distress, melhor qualidade de vida e melhor bem-estar espiritual.

Palavras-chave: espiritualidade, religião, neoplasias, qualidade de vida

\begin{abstract}
The present study aimed to evaluate the relationship between distress, quality of life, and Spirituality and Religiosity (RS) in patients newly diagnosed with cancer, in the first line of chemotherapy treatment. This is a cross-sectional study, which assessed 100 patients for distress (Distress Thermometer), quality of life (Functional Assessment of Chronic Illness Therapy - General), and spirituality (Functional Assessment of Chronic Illness Therapy - Spiritual Well-Being Scale and Duke University Religion Index). The results suggest that the RS is an important strategy to coping with diagnosis and treatment of cancer, being associated with a significantly lower level of distress, a better quality of life, and greater spiritual well-being.
\end{abstract}

Keywords: spirituality, religion, neoplasms, quality of life

\section{Resumen}

El presente estudio tuvo el objetivo de evaluar la relación entre el distress, la calidad de vida y la práctica espiritual y religiosa (ER) en pacientes con cáncer recién diagnosticados en la primera línea de tratamiento de quimioterapia. Se trata de un estudio transversal, que evaluó a 100 pacientes en cuanto al distress (Termómetro de Distress), calidad de vida (Functional Assessment of Chronic Illness Therapy - General) y espiritualidad (Functional Assessment of Chronic Illness Therapy - Spiritual Well-Being Scale y Escala de Religiosidad de la Universidad de Duke). Los resultados sugieren que el ER es una importante estrategia en el enfrentamiento del diagnóstico y tratamiento del cáncer, estando asociado significativamente a un menor nivel de distress, mejor calidad de vida y mejor bienestar espiritual.

Palabras clave: espiritualidad, religión, neoplasias, calidad de vida

\footnotetext{
${ }^{1}$ Endereço de contato: 1500 Duarte Rd., Duarte, Califórnia, Estados Unidos da América. ZIP Code: 91010. Telefone: +1 (626) 416-9660. E-mail: crisbergerot@gmail.com
} 


\section{Introdução}

Para o triênio de 2020-2022, estima-se 645 mil novos casos de câncer a cada ano, sendo próstata (homens) e mama (mulheres) os mais frequentes (Instituto Nacional de Câncer José Alencar Gomes da Silva, 2019). Independentemente do tipo de câncer, o adoecimento e o tratamento oncológico acarretam inúmeras mudanças na vida do paciente e de seus familiares, com consequente impacto na saúde física, no bem-estar emocional e no funcionamento social e familiar (Adler \& Page, 2008; Decat \& Araujo, 2010). Esse fato vem ganhando importância e reconhecimento internacional, desde a publicação do protocolo para manejo do distress em 1999, pela National Comprehensive Cancer Network (NCCN) (Holland et al., 2013). Esse termo foi escolhido por caracterizar a “. . . experiência emocional desagradável multifatorial, de natureza psicológica, social e/ou espiritual, que pode interferir na habilidade de lidar efetivamente com o câncer, os sintomas físicos e o tratamento" (Decat, Laros, \& Araujo, 2009, p. 254), advinda do enfrentamento do diagnóstico e tratamento desta doença (Holland et al., 2013). Desde então, a avaliação biopsicossocial vem sendo recomendada por diferentes instituições e organizações, assegurando que os principais domínios - psicossocial, prático e físico - sejam avaliados em momentos predefinidos do tratamento, por meio de instrumentos validados para essa finalidade (Bultz, Loscalzo, \& Clark, 2012).

Outro aspecto que vem sendo considerado na avaliação biopsicossocial é a prática espiritual e religiosa (ER); neste estudo, os conceitos de espiritualidade e religiosidade serão utilizados como termos de significados próximos, não separáveis, os quais são definidos como a busca e a conexão com uma força que é transcendente e benéfica, a qual pode ser caracterizada como Deus, Alá, HaShem, Brahman, Buda, Dao, Força Superior, Verdade, nas diversas concepções existentes nas culturas ocidentais e orientais (Koenig, 2012). A busca e a identificação por essa força transcendente são denominadas de Religiosidade Intrínseca, realizada em atividades individuais ou em grupos e organizações (Koenig \& Büssing, 2010; Koenig, 2012). Sabe-se que a prática ER auxilia o paciente no enfrentamento de qualquer adoecimento (Hill \& Pargament, 2008). Koenig (2001), por exemplo, observou que indivíduos que reportam uma maior prática religiosa costumam apresentar menos sintomas de ansiedade e de depressão; menor taxa de suicídio; melhor bem-estar psicológico; sentimento de esperança e otimismo; e maior sentido e propósito de vida. Ademais, a prática ER é comumente associada a comportamentos sociais saudáveis, como convivência social e estilo de vida que evitam situações de risco (ex.: uso de drogas ilícitas e lícitas, excesso de velocidade em condução de veículos e comportamento sexual de risco) (Koenig, 2012).

Em Oncologia, diversos autores reforçam a importância da inserção da espiritualidade e religiosidade na avaliação biopsicossocial, para uma melhor compreensão acerca da adaptação psicológica ao câncer (Jimenez-Fonseca et al., 2018; Nejat, Whitehead, \& Crowe, 2017). Sabe-se que muitos pacientes recorrem à religião e à espiritualidade no enfrentamento da doença (Bergerot et al., 2016; Gobatto \& Araujo, 2013; Iwatani, Matsuda, Kawabata, Miura, \& Matsushima, 2013). Dessa forma, pontua-se que a espiritualidade/religiosidade impactaria positivamente no enfrentamento das diferentes etapas de adoecimento em câncer (ex.: diagnóstico, fim da vida) e no entendimento do prognóstico, tornando o paciente mais ativo e participativo na tomada de decisão acerca da própria saúde (Jimenez-Fonseca et al., 2018; Whitford \& Olver, 2012). 
Camargos, Paiva, Barroso, Carneseca e Paiva (2015) reportaram que 99,2\% dos pacientes referem utilizar a prática ER durante o tratamento. Esse uso está associado a menores índices de ansiedade e de depressão, maior bem-estar emocional e maiores escores de qualidade de vida (Camargos et al., 2015; Salsman et al., 2015). Os pacientes em cuidado paliativo, por exemplo, referem uma maior percepção e aceitação do próprio estado de terminalidade (Breitbart et al., 2010; Lai et al., 2017; López-Sierra \& Rodríguez-Sánchez, 2015). Contudo alguns resultados contraditórios também são descritos, associando prática ER a uma baixa adesão aos tratamentos prescritos e, mesmo, a um atraso no tratamento (Koenig, 2001). Outras implicações negativas estão relacionadas ao sentimento de culpa, vergonha, medo, controle social excessivo e avaliação negativa de eventos adversos (Koenig, 2001; Koenig, 2012).

Entretanto as limitações existentes nos desenhos das pesquisas, a falta de controle de variáveis confundidoras no planejamento estatístico e as diferentes definições propostas para espiritualidade e religiosidade inviabilizam uma avaliação comparativa dos resultados (Gonçalves, Lucchetti, Menezes, \& Vallada, 2017; López-Sierra \& Rodríguez-Sánchez, 2015). Ademais, a não publicação de resultados negativos ou nulos favorece uma leitura tendenciosa, e o fato de a maioria desses estudos terem sido conduzidos nos Estados Unidos reforça algumas contradições existentes, em função dos resultados estarem restritos ao contexto religioso-cultural da amostra estudada (Movafagh et al., 2017; Salsman et al., 2015). No Brasil, por exemplo, uma recente revisão integrativa da literatura identificou apenas quatro estudos realizados com uma amostra brasileira de pacientes com câncer em quimioterapia (Sousa et al., 2017). Nesses estudos, a espiritualidade se mostrou uma importante estratégia no enfrentamento do câncer (Caldeira, Carvalho, \& Vieira, 2014; Mesquita et al., 2013).

Devido ao crescente interesse no domínio espiritual, nas contradições existentes no meio científico e com o intuito de ampliar a compreensão acerca dos pacientes brasileiros com câncer, o presente estudo tem o objetivo de avaliar a relação entre o distress, a qualidade de vida e o uso da prática ER em pacientes recém-diagnosticados com câncer, na fase inicial do tratamento quimioterápico. Conforme as limitações descritas na literatura, optou-se por utilizar instrumentos recomendados e validados, bem como testes estatísticos que permitem maior controle de variáveis confundidoras (ex.: tipo de câncer e estadiamento). A hipótese de o uso da prática ER estar associado a uma menor prevalência de distress e melhor qualidade de vida foi considerada.

\section{Método}

Trata-se de um estudo transversal, descritivo e quantitativo, realizado com uma amostra de conveniência. A coleta de dados ocorreu entre julho de 2016 e março de 2017, nos ambulatórios de oncologia e quimioterapia da Universidade Federal de São Paulo/Hospital São Paulo (UNIFESP/HSP).

\section{Participantes}

Um total de 106 pacientes oncológicos foram convidados a participar do presente estudo. Os critérios de elegibilidade incluíram pacientes maiores de 18 anos, recém-diagnosticados e em primeira linha de tratamento quimioterápico. Considerou-se como critério de exclusão 
pacientes que recusaram participar, aqueles com histórico prévio de algum transtorno psiquiátrico ou com algum deficit cognitivo documentado no prontuário. Seis pacientes foram excluídos; quatro apresentavam histórico de transtorno psiquiátrico, um se recusou a participar e outro desistiu durante a aplicação dos questionários. A amostra final totalizou 100 pacientes.

\section{Procedimento}

\section{Coleta de dados}

Com base nos critérios de elegibilidade, os pacientes foram contatados consecutivamente pelos psicólogos residentes do programa de Residência Multiprofissional em Oncologia da UNIFESP, na sala de espera do ambulatório da oncologia. Após esclarecimento do objetivo e da metodologia do estudo, havendo a concordância em participar, solicitava-se a assinatura do Termo de Consentimento Livre e Esclarecido (TCLE), com posterior aplicação assistida dos instrumentos que avaliaram o distress, a qualidade de vida e a prática ER. A duração média de cada avaliação foi de 35 minutos.

\section{Análise dos dados}

Uma análise descritiva das variáveis sociodemográficas e clínicas foi calculada, sendo consideradas a frequência e as medidas de tendência central e de dispersão (média e desvio-padrão). Em virtude do objetivo do presente estudo, realizou-se inicialmente a correlação entre os dados sociodemográficos e os resultados obtidos em cada instrumento Termômetro de Distress (TD), Functional Assessment of Chronic Illness Therapy - General (FACT-G), Functional Assessment of Chronic Illness Therapy - Spiritual Well-Being Scale (FACT-Sp) e Escala de Religiosidade de Duke (DUREL). Na sequência, a modelagem linear foi utilizada para identificação dos potenciais preditores dos instrumentos de medidas que avaliaram distress, qualidade de vida e a prática ER. Os preditores com pontuação inferior a 0,4 foram desconsiderados, e as variáveis confundidoras - tipo de câncer e estadiamento -, controladas. Após essa seleção, testou-se a associação entre cada instrumento (variável dependente) por meio da regressão linear (stepwise). Nesses dois últimos testes, quatro modelos foram avaliados, assumindo como variável dependente um dos instrumentos (TD, FACT-G, FACT-Sp e DUREL), e como independente, ou preditor, as variáveis sociodemográfica e demais instrumentos não considerados como variável de interesse do modelo. Para todas as análises, utilizou-se o software Statistical Package for the Social Sciences (SPSS 22.0).

\section{Instrumentos}

Questionário sociodemográfico e clínico: averiguou idade, estado civil, religião, escolaridade, raça e renda. As informações clínicas (diagnóstico e estadiamento) foram coletadas no prontuário eletrônico do paciente.

Termômetro de Distress (TD): proposto pela NCCN (Holland et al., 2013), adaptado e validado para o Português por Decat et al. (2009). Composto por uma escala que avalia o nível de distress, de 0 (sem distress) a 10 (distress extremo), e por uma Lista de Problemas (LP), com 35 itens que norteiam as possíveis causas do distress, estando ela relacionada a 
questões práticas, familiares, emocionais, espirituais e físicas. O escore maior ou igual a quatro no TD é indicativo de distress moderado a severo.

Functional Assessment of Chronic IIlness Therapy - General (FACT-G): desenvolvido para mensurar a qualidade de vida em pacientes adultos com câncer, foi traduzido e validado para o Português por Arnold et al. (2000). Composto por 27 itens que avaliam quatro domínios em uma escala Likert de 4 pontos: Bem-Estar Físico, Social/Familiar, Emocional e Funcional. Quanto maior a pontuação, melhor a qualidade de vida.

Functional Assessment of Chronic Illness Therapy - Spiritual Well-Being Scale (FACT-Sp): desenvolvido para avaliar o Bem-Estar Espiritual, foi traduzido para o Português por Bredle, Salsman, Debb, Arnold e Cella (2011) e validado por Lucchetti, Lucchetti, Gonçalves e Vallada (2015). Contém 12 itens que avaliam, em uma escala Likert de 4 pontos, o Bem-Estar Espiritual, por meio das subescalas de Sentido, Paz e Fé. Quanto maior a pontuação, maior o Bem-Estar Espiritual.

Escala de Religiosidade de Duke (DUREL): elaborada por Koenig, Parkerson e Meador (1997), adaptada e validada para o Português por Taunay et al. (2012). Composta por cinco questões que avaliam, em uma escala Likert de 5 pontos, a espiritualidade e religiosidade em três dimensões: Religiosidade Organizacional (RO), Religiosidade Não Organizacional (RNO) e Religiosidade Intrínseca (RI). A RO identifica as atividades espirituais e/ou religiosas sociais (encontros religiosos, missas ou cultos), a RNO mensura a prática religiosa de forma individual (leitura da Bíblia, rezas e orações), e a RI, a crença em valores espirituais e religiosos, o esforço individual para a vivência e internalização desses conceitos. Os três domínios são avaliados de forma independente; quanto maior a pontuação, maior a prática religiosa.

\section{Considerações éticas}

O presente estudo teve aprovação pelo Comitê de Ética em Pesquisa, da Universidade Federal de São Paulo (UNIFESP), sob o protocolo n. 600/2015.

\section{Resultados}

\section{Descrição da amostra e dos resultados obtidos nos instrumentos de medida}

Os pacientes tinham em média 51,9 anos, Desvio Padrão $(D P)=16,6$, sendo a maioria do sexo feminino (62\%), casada (52\%) e com baixa escolaridade (51\% cursou até 9 anos de ensino) (Tabela 1). A fonte de renda dos pacientes era proveniente da aposentadoria ou de benefício por incapacidade (65\%) e, em 59\% dos casos, alcançava um valor correspondente a três salários mínimos (Tabela 1). Quanto à religião, sobressaíram os católicos (44\%) e evangélicos (38\%). Os cânceres de mama (28\%) e gastrointestinal (17\%) foram os mais prevalentes, sendo que $64 \%$ foram diagnosticados em estádio avançado (III e IV). 
Tabela 1

Características sociodemográficas da amostra estudada ( $N=100)$

\begin{tabular}{|c|c|}
\hline Características & $\mathbf{N}(\%) / M(D P)$ \\
\hline \multicolumn{2}{|l|}{ Idade } \\
\hline Mín. - Máx & $18-85$ \\
\hline $\mathrm{M}(\mathrm{DP})$ & $51,9(16,6)$ \\
\hline \multicolumn{2}{|l|}{ Sexo } \\
\hline Feminino & 62 (62\%) \\
\hline Masculino & 48 (48\%) \\
\hline \multicolumn{2}{|l|}{ Estado civil } \\
\hline Solteiro & $27(27 \%)$ \\
\hline Casado & $53(53 \%)$ \\
\hline Divorciado & 10 (10\%) \\
\hline Viúvo & $9(9 \%)$ \\
\hline Ausente & $1(\%)$ \\
\hline \multicolumn{2}{|l|}{ Escolaridade } \\
\hline Analfabeto & 4 (4\%) \\
\hline Ensino Fundamental & 47 (47\%) \\
\hline Ensino Médio & $31(31 \%)$ \\
\hline Ensino Superior & $18(18 \%)$ \\
\hline \multicolumn{2}{|l|}{ Raça } \\
\hline Branca & $56(56 \%)$ \\
\hline Negra & $16(16 \%)$ \\
\hline Parda & $22(22 \%)$ \\
\hline Oriental & $4(4 \%)$ \\
\hline \multicolumn{2}{|l|}{ Ocupação } \\
\hline Estudante & $2(2 \%)$ \\
\hline Desempregado & $10(10 \%)$ \\
\hline Autônomo ou empregado & $13(13 \%)$ \\
\hline Aposentado ou afastado & $65(65 \%)$ \\
\hline Outros & $10(10 \%)$ \\
\hline \multicolumn{2}{|l|}{ Renda salarial mensal } \\
\hline Sem renda & $23(23 \%)$ \\
\hline Até 3 salários mínimos & $59(59 \%)$ \\
\hline De 3 a 5 salários mínimos & $13(13 \%)$ \\
\hline De 5 a 8 salários mínimos & $2(2 \%)$ \\
\hline Superior a 8 salários mínimos & $3(3 \%)$ \\
\hline \multicolumn{2}{|l|}{ Religião } \\
\hline Católica & $44(44 \%)$ \\
\hline Evangélica & $38(38 \%)$ \\
\hline Sem religião & $8(8 \%)$ \\
\hline Outras & $3(3 \%)$ \\
\hline \multicolumn{2}{|l|}{ Tipo de câncer } \\
\hline Mama & $28(28 \%)$ \\
\hline Gastrointestinal & $17(17 \%)$ \\
\hline Geniturinário & $12(12 \%)$ \\
\hline Sistema Nervoso Central & $9(9 \%)$ \\
\hline Cabeça e pescoço & $8(8 \%)$ \\
\hline
\end{tabular}




\begin{tabular}{lc}
\hline \multicolumn{1}{c}{ Características } & $\mathbf{N}(\%) / \mathbf{M}(\mathbf{D P})$ \\
\hline Hematológico & $7(7 \%)$ \\
Pulmão & $7(7 \%)$ \\
Outros & $12(12 \%)$ \\
Estadiamento & \\
I - II & $32(32 \%)$ \\
II - IV & $64(64 \%)$ \\
Ausente & $2(2 \%)$ \\
\hline
\end{tabular}

Distress moderado a severo foi reportado por $40 \%$ dos pacientes, Média $(M)=3,31$, $(\mathrm{DP}=2,89)$. Problemas financeiros (28\%), nervosismo (42\%), tristeza (36\%), preocupação (55\%), perda do interesse em atividades usuais (22\%), aparência (34\%), alimentação (26\%), fadiga (41\%) e dormir (35\%) foram os mais comumente citados. A média de qualidade de vida dos pacientes foi de 81,6 ( $\mathrm{DP}=15,7)$, sendo a maior média pontuada nos domínios Bem-Estar Físico ( $M=22 ; D P=5,3)$ e Social/Familiar $(M=21,2 ; D P=5,3)$, seguidos de Bem-Estar Emocional $(M=19,8 ; D P=3,7)$ e Funcional $(M=18,8 ; D P=5,4)$. Na escala Bem-Estar Espiritual, 38,7 ( $\mathrm{DP}=7,3)$ foi o escore médio obtido na FACT-Sp; 13,3 ( $\mathrm{DP}=2,2)$ na subescala Sentido; 12,6 (DP=3,1) na subescala Paz; e 12,8 (DP=2,9) na subescala Fé. No DUREL, o escore médio de espiritualidade na RI foi de 13,6 ( $D P=1,9)$; na $R O$, de 3,9 ( $D P=1,8)$; e na $R N O$, de 4,7 $(\mathrm{DP}=1,6)$. Medidos pela $\mathrm{RI}$, a crença em valores espirituais e/ou religiosos e o esforço individual para a vivência e internalização desses conceitos foram referidos por quase a totalidade da amostra (95\%). Cerca de 70\% dos participantes afirmaram realizar atividades religiosas individuais diariamente, e 49\% disseram participar de encontros semanais (Tabela 2).

Tabela 2

Índice de religiosidade reportado pelos pacientes durante o tratamento

\begin{tabular}{lc}
\hline \multicolumn{1}{c}{ Dimensões de espiritualidade e religiosidade } & $\mathbf{N}(\%)$ \\
\hline Religiosidade Intrínseca (crença em valores espirituais e religiosos) & \\
Consideram como verdade & $95(95 \%)$ \\
Não estão certos & $4(4 \%)$ \\
Não é verdade & $1(1 \%)$ \\
Religiosidade não organizacional (práticas religiosas individuais) & \\
Diariamente & $72(72 \%)$ \\
Semanalmente & $12(12 \%)$ \\
Mensalmente & $5(5 \%)$ \\
Nunca & $11(11 \%)$ \\
Religiosidade organizacional (participação de encontros religiosos) & \\
Semanalmente & $49(49 \%)$ \\
Mensalmente & $15(15 \%)$ \\
Anualmente & $17(17 \%)$ \\
Nunca & $19(19 \%)$ \\
\hline
\end{tabular}

\section{Relação entre o distress, a qualidade de vida e a espiritualidade e religiosidade}

Idade jovem esteve associada significativamente a um maior escore de distress ( $r=-0,62$; $p=0,02)$, maior RI $(r=-0,64 ; p=0,02)$ e RO $(r=-0,59 ; p=0,003)$. Observou-se também uma correlação entre maior escolaridade e maior escore de distress $(r=0,69 ; p=0,009)$, presença de 
sintomas depressivos $(r=0,63 ; p=0,03)$, preocupação $(r=0,67 ; p=0,007)$ e perda do interesse nas atividades usuais $(r=0,61 ; p=0,02)$. Ademais, quanto menor a renda mensal, maior foi o uso da RO ( $r=-0.60 ; p=0,002$ ). Nenhuma associação significativa foi encontrada entre os instrumentos de medida e as variáveis sexo, estado civil e raça.

Entre os instrumentos de medida, constatou-se uma associação significativa entre maior escore de distress e menor utilização da RI $(r=-0,57 ; p=0,002)$, da RNO $(r=-0,58 ; p=0,005)$ e menor Bem-Estar Espiritual ( $r=-0,67 ; p=0,000)$. Melhor qualidade de vida esteve correlacionada significativamente com a RI $(r=0,69 ; p=0,004)$, a RNO $(r=0,62 ; p=0,000)$ e com maior Bem-Estar Espiritual $(r=0,72 ; p=0,000)$. Os quatro domínios avaliados pelo FACT-G - Físico, Social/Familiar, Emocional e Funcional - estiveram também associados significativamente a um maior Bem-Estar Espiritual ( $r=0,70, r=0,76, r=0,85$ e $r=0,84$, respectivamente; $p s<0,001$ ) e utilização da RI $(r=0,65, r=0,66, r=0,55$ e $r=0,53$, respectivamente; $p s<0,05)$ e da RNO $(r=0,57, r=0,58, r=0,63$ e $r=0,60$, respectivamente; $p s<0,05)$.

A modelagem linear identificou os principais preditores dos instrumentos de medida: o distress (TD) se mostrou um importante preditor da qualidade de vida (FACT-G). Já a qualidade de vida (FACT-G) foi preditora de Bem-Estar Espiritual (FACT-SP), e o Bem-Estar Espiritual (FACT-SP), da qualidade de vida global (FACT-G), tal como descrito na Tabela 3. Na regressão linear, os fatores idade (jovem), presença de problemas emocionais e menor pontuação na subescala paz (FACT-Sp) foram preditores de um maior escore de distress. Melhor qualidade de vida esteve associada à ausência de problemas familiares, emocionais e físicos, a baixo distress e a um maior escore nas subescalas Sentido e Paz (FACT-Sp). Outrossim, a presença de problemas práticos, maior pontuação na subescala Fé, na RO e na RNO, baixo distress e ausência de sintomas físicos estiveram associados a um maior uso da RI (Tabela 4).

Tabela 3

Modelagem linear da importância do Bem-Estar Espiritual (FACT-Sp), do distress (DT) e da qualidade de vida (FACT-G)

\begin{tabular}{lc}
\hline \multicolumn{1}{c}{ Preditor } & Pontuação (0-1) \\
\hline FACT-SP & \\
FACT-G & 0,62 \\
Religiosidade Intrínseca & 0,18 \\
Bem-Estar Funcional (FACT-G) & 0,09 \\
Distress & 0,08 \\
Religiosidade não organizacional & 0,06 \\
TD & \\
FACT-G & 0,49 \\
Bem-Estar Social/Familiar (FACT-G) & 0,19 \\
FACT-Sp & 0,19 \\
Idade & 0,13 \\
FACT-G & \\
FACT-Sp & 0,77 \\
Distress & 0,16 \\
Estádio da doença & 0,08 \\
\hline
\end{tabular}


Tabela 4

Regressão linear das variáveis associadas ao distress (DT), à qualidade de vida (FACT-G) e à religiosidade (DUREL)

\begin{tabular}{|c|c|c|c|}
\hline Covariáveis & $\begin{array}{c}\text { Coeficiente B } \\
\text { (Desvio-Padrão) }\end{array}$ & Teste $-\mathrm{t}$ & Valor de $\mathrm{P}$ \\
\hline \multicolumn{4}{|l|}{ Distress (TD) } \\
\hline Idade & $-0,04(0,02)$ & $-2,45$ & 0,01 \\
\hline Problemas emocionais & $0,83(0,18)$ & 4,64 & 0,000 \\
\hline Subescala paz & $-0,32(0,13)$ & $-2,52$ & 0,01 \\
\hline \multicolumn{4}{|l|}{ Qualidade de vida (FACT-G) } \\
\hline Problemas familiares & $-5,69(2,77)$ & $-2,05$ & 0,04 \\
\hline Problemas emocionais & $-3,43(0,89)$ & $-3,84$ & 0,000 \\
\hline Problemas físicos & $-1,66(0,41)$ & -4 & 0,000 \\
\hline Distress & $-1,32(0,51)$ & $-2,59$ & 0,01 \\
\hline Subescala sentido & $1,95(0,69)$ & 2,82 & 0,06 \\
\hline Subescala paz & $1,01(0,51)$ & 1,97 & 0,05 \\
\hline \multicolumn{4}{|l|}{ Religiosidade Intrínseca (DUREL) } \\
\hline Problemas práticos & $0,41(0,19)$ & 2,16 & 0,03 \\
\hline Problemas físicos & $-0,21(0,07)$ & $-3,09$ & 0,003 \\
\hline Distress & $-0,19(0,08)$ & $-2,23$ & 0,02 \\
\hline Subescala fé & $0,49(0,07)$ & 6,91 & 0,000 \\
\hline Religiosidade organizacional & $0,25(0,08)$ & 2,81 & 0,006 \\
\hline Religiosidade não organizacional & $0,23(0,11)$ & 2,16 & 0,03 \\
\hline
\end{tabular}

\section{Discussão}

A prática ER esteve associada a um baixo distress e a uma melhor qualidade de vida, rejeitando a hipótese nula. Notavelmente, os resultados descritos no presente estudo sugerem a prática ER como um relevante recurso no enfrentamento do diagnóstico e do tratamento em pacientes oncológicos brasileiros, tratados em um hospital público de referência. Verificouse uma substancial morbidade emocional e física experienciada por esses pacientes, reforçando a utilidade de uma rotina de avaliação biopsicossocial e espiritual para identificação dos pacientes em risco que se beneficiariam de um suporte psicológico ou mesmo de um suporte espiritual; ambos considerados importantes componentes na assistência oncológica (Balboni et al., 2017).

Uma grande proporção dos pacientes reportou um distress moderado a severo, caracterizado por diversos problemas físicos, emocionais e práticos, relacionados ao impacto do diagnóstico e efeitos colaterais do tratamento. Contudo constatou-se uma discrepância entre esse resultado e os descritos na literatura nacional (Bergerot, Philip, et al., 2017) e internacional (Zabora, BrintzenhofeSzoc, Curbow, Hooker, \& Piantadosi, 2001), provavelmente, em resposta às dificuldades encontradas no serviço público brasileiro (ex.: falta de medicamento, manejo insatisfatório de sinais e sintomas). Já o escore médio de qualidade de vida esteve próximo ao da média da população americana com câncer (Brucker, Yost, Cashy, Webster, \& Cella, 2005), com discreta piora para Bem-Estar Emocional e Funcional. Ambos resultados ratificam a relevância da rotina de avaliação biopsicossocial para proposição de um planejamento terapêutico 
que atenda às necessidades específicas de cada paciente e otimize os recursos existentes nas instituições (Bergerot et al., 2016; Bergerot, Philip, et al., 2017).

Quanto aos fatores de risco biopsicossocial, apesar de gênero, estado civil e raça não estarem associados ao distress, à qualidade de vida ou à prática ER, a idade jovem esteve associada a um maior nível de distress e, curiosamente, a uma maior crença espiritual e religiosa (RI) e a uma maior prática religiosa (RO e RNO). É previsto que a população jovem adulta apresente uma maior prevalência de distress, contudo é curiosa a associação dessa faixa etária com o uso da prática ER (Zebrack, Mathews-Bradshaw, \& Siegel, 2010). Entre os poucos estudos publicados com pacientes jovens, a espiritualidade esteve associada a maiores questionamentos existenciais, estando esses pacientes mais vulneráveis ao medo, a preocupações e ao sentimento de culpa (Barton et al., 2018; Weaver \& Wratchford, 2017). Apesar dos resultados do presente estudo sugerirem que a prática ER seja utilizada como uma possivel ferramenta nas intervenções e proposições terapêuticas, esse uso precisa ser mais bem compreendido na população jovem adulta como estratégia de enfrentamento do adoecimento em câncer.

Ademais, uma maior escolaridade esteve associada a um maior escore de distress e a presença de sintomas depressivos e de ansiedade. Esse achado gera uma hipótese e sugere uma possível relação entre a compreensão acerca da gravidade do próprio diagnóstico, contribuindo para a presença de sintomas emocionais. Até o momento, nenhum estudo avaliou a influência dessa compreensão na prevalência de sintomas de ansiedade e/ou de depressão, ou mesmo na utilização da prática ER. Recomenda-se a condução de novos estudos que explorem a relação entre o distress, a prática ER e o entendimento do prognóstico, haja vista a tradição cultural da população brasileira em atribuir à doença e ao sucesso do tratamento um componente espiritual (Laplantine, 2001; Mello \& Oliveira, 2013).

Por fim, observou-se que a maioria dos pacientes atestaram crer em valores religiosos e espirituais, reforçando a relevância da prática ER como um recurso no enfrentamento do câncer. A utilização da prática ER esteve associada a menores escores de distress, ausência de problemas familiares e melhor qualidade de vida, sugerindo um possível efeito protetor na vivência do câncer (Camargos, et al., 2015; Salsman et al., 2015). Para alguns pesquisadores, esse desfecho pode ser advindo do aumento da sensação de controle proporcionado pela prática religiosa individual (Esperandio \& Ladd, 2013). Há ainda a presença do sentido e do propósito de vida provenientes do uso da prática ER, contribuindo para um melhor enfrentamento dos desafios inerentes a essa doença (Aquino et al., 2009; Breitbart et al., 2012; Koenig, 2001). Todavia o uso da prática ER precisa ser mais bem entendido no contexto de atrasos e recusas na adesão a tratamentos convencionais. Na circunstância em que a espiritualidade implica um tratamento espiritual (não mensurado nesta pesquisa), um estudo recente demonstrou que o uso do tratamento alternativo em detrimento do convencional impacta negativamente na sobrevida de pacientes com câncer (Johnson, Park, Gross, \& Yu, 2018). Curiosamente, o uso dessas terapias foi maior em pacientes com idade jovem, maior escolaridade, alto nível socioeconômico e diagnosticados em estádio avançado da doença (Johnson et al., 2018).

O presente estudo amplia alguns resultados já descritos, sugerindo um potencial benefício do uso da prática ER como uma estratégia adaptativa ao câncer. Porém algumas limitações precisam ser consideradas para a generalização dos resultados encontrados, tais como 
o tamanho restrito e a não homogeneidade da amostra. Recomendam-se novas pesquisas que ampliem a amostra e que entendam as diferenças no uso da prática ER para cada tipo de câncer, ou mesmo estadiamento clínico/patológico. Há, ainda, o fato de esta pesquisa ter sido conduzida em apenas um serviço público de Oncologia. Ademais, este estudo não avaliou outras estratégias de enfrentamento ou mesmo as possíveis mudanças no uso dessas estratégias nas diferentes fases de adoecimento em câncer. Novos estudos que contemplem outras instituições e o impacto longitudinal dessas estratégias de enfrentamento no distress e na qualidade de vida precisam ser propostos, auxiliando a decifrar os possíveis mecanismos subjacentes ao enfrentamento do câncer.

\section{Conclusão}

A prática ER mostrou ser uma importante estratégia no enfrentamento do diagnóstico e tratamento do câncer. A prática ER esteve associada significativamente a menor nível de distress, maior qualidade de vida e maior bem-estar espiritual. Idade jovem e maior escolaridade foram fatores de risco de distress. Esses achados destacam a relevância de uma melhor compreensão acerca dos benefícios da prática ER, como uma ferramenta para o planejamento terapêutico, auxiliando o paciente no enfrentamento do câncer. Futuras pesquisas devem explorar o benefício de intervenções focadas na prática ER, bem como a avaliação de sua efetividade na redução do nível de distress e na melhora da qualidade de vida em diferentes cenários da vivência do câncer.

\section{Referências}

Adler, N. E., \& Page, A. E. K. (Eds.). (2008). Cancer care for the whole patient: Meeting psychosocial health needs. Washington: National Academies Press. doi:https://doi. org/10.17226/11993

Arnold, B. J., Eremenco, S., Chang, C. H., Odom, L., Ribaudo, J., \& Cella, D. (2000). Development of a single Portuguese language version of the Functional Assessment of Cancer TherapyGeneral (FACT-G) Scale. Quality of Life Res earch, 9(3), 316. doi:https://doi.org/10.1023/A:1017291624003

Aquino, T. A. A. D., Correia, A. P. M., Marques, A. L. C., Souza, C. G. D., Assis Freitas, H. C. D., Araújo, I. F. D., . . Araújo, W. F. D. (2009). Religious attitude and the meaning of life: a correlational study. Psicologia: Ciência e Profissão, 29(2), 228-243. doi:https://doi. org/10.1590/S1414-98932009000200003

Balboni, T. A., Fitchett, G., Handzo, G. F., Johnson, K. S., Koenig, H. G., Pargament, K. I., . . . Steinhauser, K. E. (2017). State of the Science of Spirituality and Palliative Care Research Part II: Screening, Assessment, and Interventions. Journal of Pain and Symptom Management, 54(3), 441-453. doi:https://doi.org/10.1016/j.jpainsymman.2017.07.029

Barton, K. S., Tate, T., Lau, N., Taliesin, K. B., Waldman, E. D., \& Rosenberg, A. R. (2018). “I'm not a spiritual person". How hope might facilitate conversations about spirituality among teens and young adults with cancer. Journal of Pain and Symptom Management, 55(6), 1599-1608. doi:https://doi.org/10.1016/j.jpainsymman.2018.02.001

Bergerot, C. D., Philip, E. J., Schuler, T. A., Clark, K. L., Loscalzo, M., Buso, M. M., . . A Araujo, T. C. C. F. (2016). Development and implementation of a comprehensive psychosocial 
screening program in a Brazilian cancer center. Psycho-Oncology, 25(11), 1343-1349. doi:https://doi.org/10.1002/pon.4275

Bergerot, C. D., Philip, E. J., Zayat, C. G., Azevedo, I. M. D., Araujo, T. C. C. F. D., \& De Domenico, E. B. L. (2017). Investigating the two-tiered system of psychosocial cancer care in Brazil using a distress screening measure. Journal of Global Oncology, 3(1), 1-6. doi:https://doi. org/10.1200/JGO.2016.004978

Bergerot C. D., Zayat C. G., Azevedo I. M., Okuma G. Y., Pedras R. N., Manhães M. F. M., \& Domenico, E. B. L. (2017). Implementação de rotina de psico-oncologia segundo recomendações internacionais aplicadas em serviço público brasileiro. Estudos de Psicologia (UFRN), 22(4), 350-357. doi:http://dx.doi.org/10.22491/1678-4669.20170036

Bredle, J. M., Salsman, J. M., Debb, S. M., Arnold, B. J., \& Cella, D. (2011). Spiritual well-being as a component of health-related quality of life: the Functional Assessment of Chronic Illness Therapy-Spiritual Well-Being Scale (FACIT-Sp). Religions, 2(1), 77-94. doi:https:// doi.org/10.3390/rel2010077

Breitbart, W., Poppito, S., Rosenfeld, B., Vickers, A. J., Li, Y., Abbey, J., . . . Cassileth, B. R. (2012). Pilot randomized controlled trial of individual meaning-centered psychotherapy for patients with advanced cancer. Journal of Clinical Oncology, 30(12), 1304-1309. doi:https://doi.org/10.1200/JCO.2011.36.2517

Breitbart, W., Rosenfeld, B., Gibson, C., Pessin, H., Poppito, S., Nelson, C., . . Sorger, B. (2010). Meaning-centered group psychotherapy for patients with advanced cancer: A pilot randomized controlled trial. Psycho-Oncology, 19(1), 21-28. doi:https://doi.org/10.1002/ pon.1556

Brucker, P. S., Yost, K., Cashy, J., Webster, K., \& Cella, D. (2005). General population and cancer patient norms for the Functional Assessment of Cancer Therapy-General (FACT-G). Evaluation \& The Health Professions, 28(2), 192-211. doi:https://doi. org/10.1177/0163278705275341

Bultz, B. D., Loscalzo, M. J., \& Clark, K. L. (2012). Screening for distress, the 6th vital sign, as the connective tissue of health care systems: a roadmap to integrated interdisciplinary person-centered care. In L. Grassi \& M. Riba (Eds.), Clinical Psycho-Oncology: An International Perspective (pp. 83-96). Oxford, UK: John Wiley \& Sons Ltd. doi:https://doi. org/10.1002/9781119941101.ch7

Caldeira, S., Carvalho, E. C. D., \& Vieira, M. (2014). Between spiritual wellbeing and spiritual distress: Possible related factors in elderly patients with cancer. Revista Latino-Americana de Enfermagem, 22(1), 28-34. doi:https://doi.org/10.1590/0104-1169.3073.2382

Camargos, M. G., Paiva, C. E., Barroso, E. M., Carneseca, E. C., \& Paiva, B. S. R. (2015). Understanding the differences between oncology patients and oncology health professionals concerning spirituality/religiosity: A cross-sectional study. Medicine, 94(47), 1-15. doi:https://doi.org/10.1097/MD.0000000000002145

Decat, C. S., \& Araujo, T. C. C. F. D. (2010). Psico-oncologia: Apontamentos sobre a evolução histórica de um campo interdisciplinar. Brasília Médica, 47(1), 93-99.

Decat, C. S. A., Laros, J. A., \& Araujo, T. C. C. F. D. (2009). Termômetro de distress: validação de um instrumento breve para avaliação diagnóstica de pacientes oncológicos. PsicoUSF, 14(3), 253-260. doi:https://doi.org/10.1590/\$1413-82712009000300002 
Esperandio, M. R. G., \& Ladd, K. L. (2013). Oração e saúde: Questões para a Teologia e para a Psicologia da Religião (Prayer and Health: Issues for theology and psychology of religion). Horizonte, 11(30), 627-656. doi:https://doi.org/10.5752/P.2175-5841.2013v11n30p627

Gobatto, C. A., \& Araujo, T. C. C. F. D. (2013). Religiosidade e espiritualidade em oncologia: Concepções de profissionais da saúde. Psicologia Universidade de São Paulo, 24(1), 1134. doi:https://doi.org/10.1590/S0103-65642013000100002

Gonçalves, J. P. B., Lucchetti, G., Menezes, P. R., \& Vallada, H. (2017). Complementary religious and spiritual interventions in physical health and quality of life: $A$ systematic review of randomized controlled clinical trials. Plos One, 12(10), e0186539. doi:https:// doi.org/10.1371/journal.pone.0186539

Hill, P. C., \& Pargament, K. I. (2008). Advances in the conceptualization and measurement of religion and spirituality: Implications for physical and mental health research. Psychology of Religion and Spirituality, S(1), 3-17. doi:https://doi.org/10.1037/1941-1022.S.1.3

Holland, J. C., Andersen, B., Breitbart, W. S., Buchmann, L. O., Compas, B., Deshields, T. L., ... Greiner, C. B. (2013). Distress management. Journal of the National Comprehensive Cancer Network, 11(2), 190-209. doi:https://doi.org/10.6004/jnccn.2013.0027

Instituto Nacional de Câncer José Alencar Gomes da Silva. (2019). Estimativa 2020: Incidência de câncer no Brasil. Recuperado de https://www.inca.gov.br/sites/ufu.sti.inca.local/files// media/document//estimativa-2020-incidencia-de-cancer-no-brasil.pdf

Iwatani, T., Matsuda, A., Kawabata, H., Miura, D., \& Matsushima, E. (2013). Predictive factors for psychological distress related to diagnosis of breast cancer. Psycho-Oncology, 22(3), 523-529. doi:https://doi.org/10.1002/pon.3023

Jimenez-Fonseca, P., Lorenzo-Seva, U., Ferrando, P. J., Carmona-Bayonas, A., Beato, C., García, T., ... Calderon, C. (2018). The mediating role of spirituality (meaning, peace, faith) between psychological distress and mental adjustment in cancer patients. Supportive Care in Cancer, 26(5), 1411-1418. doi:https://doi.org/10.1007/s00520-017-3969-0

Johnson, S. B., Park, H. S., Gross, C. P., \& Yu, J. B. (2018). Use of alternative medicine for cancer and its impact on survival. JNCl: Journal of the National Cancer Institute, 110(1), 121-124. doi:https://doi.org/10.1093/jnci/djx145

Koenig, H. G. (2001). Religion and medicine II: Religion, mental health, and related behaviors.

The International Journal of Psychiatry in Medicine, 31(1), 97-109. doi:https://doi. org/10.2190/BK1B-18TR-X1NN-36GG

Koenig, H. G. (2012). Religion, spirituality, and health: The research and clinical implications. ISRN psychiatry, 2012(278730), 1-33. doi: https://doi.org/10.5402/2012/278730

Koenig, H. G., \& Büssing, A. (2010). The Duke University Religion Index (DUREL): a fiveitem measure for use in epidemiological studies. Religions, 1(1), 78-85. doi:https://doi. org/10.3390/rel1010078

Koenig, H. G., Parkerson, G. R., \& Meador, K. G. (1997). Religion index for psychiatric research. American Journal of Psychiatry, 154(6), 885-886. doi:https://doi.org/10.1176/ ajp.154.6.885b

Lai, C., Luciani, M., Galli, F., Morelli, E., Del Prete, F., Ginobbi, P., . . Lombardo, L. (2017). Spirituality and awareness of diagnoses in terminally ill patients with cancer. American Journal of Hospice and Palliative Medicine, 34(6), 505-509. doi:https://doi. org/10.1177/1049909116630985 
Laplantine, F. (2001). Antropologia dos sistemas de representações da doença: Sobre algumas pesquisas desenvolvidas na França contemporânea reexaminadas à luz de uma experiência brasileira. In D. Jodelet (Ed.), As representações sociais (pp. 241-259). Rio de Janeiro: EDUERJ.

López-Sierra, H. E., \& Rodríguez-Sánchez, J. (2015). The supportive roles of religion and spirituality in end-of-life and palliative care of patients with cancer in a culturally diverse context: A literature review. Current Opinion in Supportive and Palliative Care, 9(1), 87-95. doi:https://doi.org/10.1097/SPC.0000000000000119

Lucchetti, G., Lucchetti, A. L. G., Gonçalves, J. P. B., \& Vallada, H. P. (2015). Validation of the Portuguese version of the Functional Assessment of Chronic Illness Therapy-Spiritual Well-Being scale (FACIT-Sp 12) among Brazilian psychiatric inpatients. Journal of Religion and Health, 54(1), 112-121. doi:https://doi.org/10.1007/s10943-013-9785-z

Mello, M. L., \& Oliveira, S. S. (2013). Health, religion and culture: A dialogue based on AfroBrazilian customs. Saúde e Sociedade, 22(4), 1024-1035. doi:https://doi.org/10.1590/ S0104-12902013000400006

Mesquita, A. C., Chaves, É. D. C. L., Avelino, C. C. V., Nogueira, D. A., Panzini, R. G., \& Carvalho, E. C. D. (2013). The use of religious/spiritual coping among patients with cancer undergoing chemotherapy treatment. Revista Latino-Americana de Enfermagem, 21(2), 539-545. doi:https://doi.org/10.1590/S0104-11692013000200010

Movafagh, A., Heidari, M. H., Abdoljabbari, M., Mansouri, N., Taghavi, A., Karamatinia, A., . . Ghazi, M. (2017). Spiritual Therapy in Coping with Cancer as a Complementary Medical Preventive Practice. Journal of Cancer Prevention, 22(2), 82. doi:https://doi.org/10.15430/ JCP.2017.22.2.82

Nejat, N., Whitehead, L., \& Crowe, M. (2017). The use of spirituality and religiosity in coping with colorectal cancer. Contemporary Nurse, 53(1), 48-59. doi:https://doi.org/10.1080/1 0376178.2016 .1276401

Salsman, J. M., Pustejovsky, J. E., Jim, H. S., Munoz, A. R., Merluzzi, T. V., Park, C. L., . . . Fitchett, G. (2015). A meta-analytic approach to examining the correlation between religion/spirituality and mental health in cancer. Cancer, 121, 3769-3778. doi:https://doi. org/10.1002/cncr.29350

Sousa, F. F. D. P. R., Freitas, S. M. F. D. M., Farias, A. G. D. S., Cunha, M. D. C. D. S., Araújo, M. F. M. D., \& Veras, V. S. (2017). Enfrentamento religioso/espiritual em pessoas com câncer em quimioterapia: revisão integrativa da literatura. SMAD: Revista Eletrônica Saúde Mental Álcool e Drogas, 13(1), 45-51. doi:https://doi.org/10.11606/issn.1806-6976.v13i1p45-51

Taunay, T. C. D., Gondim, F. D. A. A., Macêdo, D. S., Moreira-Almeida, A., Gurgel, L. D. A., Andrade, L. M. S., \& Carvalho, A. F. (2012). Validação da versão brasileira da escala de religiosidade de Duke (DUREL). Revista de Psiquiatria Clínica, 39(4), 130-135. doi:https:// doi.org/10.1590/S0101-60832012000400003

Weaver, M. S., \& Wratchford, D. (2017). Spirituality in adolescent patients. Annals of Palliative Medicine, 6(3), 270-278. doi:https://doi.org/10.21037/apm.2017.05.09

Whitford, H. S., \& Olver, I. N. (2012). The multidimensionality of spiritual wellbeing: Peace, meaning, and faith and their association with quality of life and coping in oncology. Psycho-Oncology, 21(6), 602-610. doi:https://doi.org/10.1002/pon.1937 
Zabora, J., BrintzenhofeSzoc, K., Curbow, B., Hooker, C., \& Piantadosi, S. (2001). The prevalence of psychological distress by cancer site. Psycho-Oncology, 10(1), 19-28. doi:https://doi. org/10.1002/1099-1611(200101/02)10:1<19::AID-PON501>3.0.CO;2-6

Zebrack, B., Mathews-Bradshaw, B., \& Siegel, S. (2010). Quality cancer care for adolescents and young adults: A position statement. Journal of Clinical Oncology, 28(32), 4862-4867. doi:https://doi.org/10.1200/JCO.2010.30.5417

Recebido em: 21/08/2019

Última revisão: 23/10/2019

Aceite final: 26/11/2019

\section{Sobre os autores:}

Getulio Yuzo Okuma: Especialista em Psicologia da Saúde pela Universidade Federal de São Paulo (UNIFESP). Graduado em Psicologia pela Universidade Mogi das Cruzes (UMC). Psicólogo na Rede de Reabilitação Lucy Montoro. E-mail: getulio.okuma@gmail.com, Orcid: http://orcid.org/0000-0001-6436-2607

MariaFernanda MarcussoManhães: Especialistaem Psicologia daSaúdeeHospitalarpela Universidade Federal de São Paulo (UNIFESP). Graduada em Psicologia pela Universidade Federal de Santa Catarina (UFSC).Psicóloga do grupo Oncologistas Associados. E-mail: manhaes.mfernanda@gmail.com, Orcid: http://orcid.org/0000-0001-9623-8950

Renata Nunes Pedras: Especialista em Psicologia da Saúde pela Universidade Federal de São Paulo (UNIFESP) e em Psicologia Hospitalar pelo Hospital das Clínicas da Faculdade de Medicina da Universidade de São Paulo (HC-FM-USP). Graduada em Psicologia pela Universidade Cruzeiro do Sul (UNICSUL). E-mail: renatanunespedras@gmail.com, Orcid: http://orcid.org/0000-0003-2716-0664

Isadora Miranda Azevedo: Especialista em Psicologia da Saúde pela Universidade Federal de São Paulo (UNIFESP) e pela Universidade Federal do Rio Grande do Norte (UFRN). Graduada em Psicologia pela UFRN. E-mail: azevedoisadora22@gmail.com, Orcid: http://orcid.org/0000-0001-7060-6356

Edvane Birelo Lopes De Domenico: Doutora em Enfermagem pela Escola de Enfermagem da Universidade de São Paulo (EEUSP). Mestre em Enfermagem pela EEUSP. Especialista em Enfermagem Oncológica pela Faculdade de Enfermagem do Hospital Israelita Albert Einstein (FEHIAE). Graduada em Enfermagem pela Universidade de São Paulo (USP). Professora associada e livre-docente na Universidade Federal de São Paulo (UNIFESP). Orientadora do programa de Mestrado Profissional do Centro de Desenvolvimento do Ensino Superior em Saúde (CEDESS) na UNIFESP. Líder do Grupo de Pesquisa GEPEBE. Tutora em Enfermagem da Residência Multiprofissional em Oncologia do Hospital São Paulo na UNIFESP. Coordenadora do programa de Extensão Universitária Acolhe-Onco: interdisciplinaridade no cuidado integral ao paciente com câncer. E-mail: domenico.edvane@unifesp.br, Orcid: http://orcid.org/0000-0001-7455-1727

Cristiane Decat Bergerot: Doutora e mestre em Processos do Desenvolvimento Humano e Saúde, com área de concentração em Psicologia da Saúde, pela Universidade de Brasília (UnB). Especialista em Psicologia da Saúde e Hospitalar pela Associação de Combate ao Câncer de Goiás (ACCG). Graduada em Psicologia pelo Centro Universitário de Brasília (UniCEUB). Coordenadora da equipe multidisciplinar do Centro de Câncer de Brasília e do Instituto Unity. Staff cientista do Instituto Unity de Ensino e Pesquisa. Pesquisadora visitante no Department of Medical Oncology \& Experimental Therapeutics, do City of Hope Comprehensive Cancer Center, Duarte, Califórnia, Estados Unidos. E-mail: crisbergerot@gmail.com, Orcid: http://orcid.org/0000-0003-0037-0303 
